\title{
Foetal alcohol syndrome: a case report from Sri Lanka
}

\author{
Chathurie Suraweera, Raveen Hanwella
}

\section{Summary}

Alcohol dependence is not a major health problem among females in Sri Lanka unlike in males. Hence the adverse effects unique to alcohol consumption in females are uncommon. Foetal alcohol syndrome, a consequence of alcohol use among females, is not commonly seen in our country. We report a case of foetal alcohol syndrome in Sri Lanka.

SL J Psychiatry 2013; 4 (2):41-42

\section{Introduction}

Alcohol is a teratogen. Foetal alcohol syndrome (FAS) is the most severe form of damage due to alcohol use in pregnancy. FAS is associated with abnormalities in the central nervous system, facial dysmorphism and growth defects. For every child born with foetal alcohol syndrome many more are born with neurobehavioural changes due to prenatal alcohol exposure. These changes are called the foetal alcohol spectrum disorder (FASD). Alcohol affects all stages of foetal development and the neurocognitive consequences are lifelong. A pregnant woman who drinks any amount of alcohol is at risk for having a child with FAS. No "safe" level of alcohol use during pregnancy has been established and larger amounts of alcohol increase the risk. Binge drinking is more harmful than drinking smaller amounts of alcohol daily. Timing of alcohol use during pregnancy is important as alcohol use is most harmful during the first three months of pregnancy; however, drinking alcohol any time during pregnancy can be harmful (1). Foetal alcohol syndrome is not common in Sri Lanka because alcohol consumption among females is low. However doctors need to be aware of it to recognise the rare cases.

\section{Case report}

The 34 year old woman we report has been dependent on alcohol since the age of 27 years. She claims to be abstinent from alcohol since getting to know of her pregnancy in March 2012. Although it is quite certain that the foetus had been exposed to alcohol, the period of exposure cannot be determined accurately as she was unaware of her last regular menstrual period. The foetus was exposed to about 5-6 units of alcohol daily as toddy. Toddy is an alcoholic beverage is made by fermenting the sap of the coconut palm. The mother had not attended antenatal care and a low birth weight pre term baby was born through normal vaginal delivery. The baby failed to thrive and had frequent respiratory tract infections. The baby was subsequently admitted with a lower respiratory tract infection. Although the mother denied use of alcohol, the admitting house officer noted that she was smelling of alcohol and therefore referred her to the psychiatry unit.

On examination the following features of foetal alcohol syndrome were present: intrauterine growth retardation, failure to thrive, decreased muscle tone, atrial septal defect, narrow small eyes with large epicanthal folds, small head, small upper jaw, smooth and thin upper lip and absent corpus callosum. The following features were not present; poor coordination, developmental delay, and smooth groove in upper lip (5).

The mother was motivated to stop alcohol. The patient did not need detoxification as she did not have withdrawal symptoms. Although it would have been best to start the patient on medication such as naltrexone or disulfiram to maintain abstinence, we did not do so because of the need to breast feed the baby with failure to thrive and the very unstable financial situation. We used cognitive behaviour therapy which focussed on managing craving and avoidance of triggers.

On discharge, the care of the patient was handed over to the community mental health team of the area for follow up and regular engagement of the patient for maintenance of abstinence. Two months after discharge, the patient remained abstinent.

\section{Discussion}

The term FAS was first used in 1973 by a group of paediatricians and psychiatrists in an article published in the Lancet (6). The syndrome however has been described earlier in 1968 by a French team (7). Prior to this alcohol was not recognised as a teratogen. In the West $30-35 \%$ pregnant women continue to consume alcohol during pregnancy. Alcohol is now the leading cause of birth defects and developmental disorders in the United States (8).

FAS and FASD consist of a distinct pattern of birth defects caused by in utero alcohol exposure. The main features of FAS include growth retardation (pre and 
postnatal), central nervous system dysfunction, and a characteristic pattern of atypical facial morphometry. The term FASD is used when there is heavy prenatal exposure to alcohol, but characteristic facial morphometry of FAS is not present. FASD commonly involves cognitive and psychosocial abnormalities consistent with FAS, including slowed information processing and impaired executive control $(9,10)$. Individuals with FASD also exhibit greater impairment with increased task complexity, in both verbal and nonverbal domains (11). Callosal malformation is common in FASD. Incidence of callosal agenesis is reportedly $6.8 \%$ in FAS, which is much higher than the incidence in the general population (about $0.025 \%$ ) (12).

FAS is unusual in Sri Lanka because of low prevalence of alcohol use among females in Sri Lanka. This case report describes an extreme form of FAS where most of the classically described features were present in the baby.

It is of utmost importance that females at risk are educated about the harm from alcohol use during pregnancy and risk of FAS. Females who intend to get pregnant should be advised to stop even social drinking as alcohol can cause subtle neurocognitive changes in the foetus even before the pregnancy is detected.

\section{Declaration of interest \\ None declared}

Chaturie Suraweera, Senior Registar in Psychiatry, University Psychiatry Unit, National Hospital of Sri Lanka, Sri Lanka

Raveen Hanwella, Professor in Psychiatry

Faculty of Medicine, University of Colombo, Sri Lanka

Corresponding author

Chaturie Suraweera

E mail:dr.chathurie@gmail.com

\section{References}

1. Carlo WA. Fetal alcohol syndrome. In Nelson Textbook of Pediatrics. 18th ed, Ed. B.R. Kliegman RM, Jenson HB, Stanton BF, eds. Vol. chap 100.2. 2007: Philadelphia, Pa: Saunders Elsevier; .

2. de Silva V, Samarasinghe D, Gunawardena N. Alcohol and Tobacco use in two districts in Sri Lanka Ceylon Medical Journal 2009;54(4): 119124

3. de Silva V, Samarasinghe D, Hanwella R. Association between alcohol and tobacco use and poverty Drug Alcohol Rev. 2011;30(1):69-73

4. Hanwella R, de Silva VA, Jayasekera NE.Alcohol use in a military population deployed in combat areas: a cross sectional study. Subst Abuse Treat Prev Policy. 2012;;7:24

5. Riley, E.P., M.A. Infante, and K.R. Warren, Fetal alcohol spectrum disorders: an overview. Neuropsychol Rev, 2011; 21(2): p. 73-80.

6. Jones, K.L. and D.W. Smith, Recognition of the fetal alcohol syndrome in early infancy. Lancet, 1973; 302(7836): p. 999-1001.

7. Lemoine, P., H. Harousseau, J. P. Borteyru, and J. C. Menuet, Les Enfants de Parents Alcooliques. Anomalies Observees a Propos de 127 Cas (Children of Alcoholic Parents: Abnormalities Observed in 127 Cases). Ouest Medica, 1968; 21: p. 476-82.

8. Bailey, B.A. and R.J. Sokol, Pregnancy and alcohol use: evidence and recommendations for prenatal care. Clin Obstet Gynecol, 2008; 51(2): 436-44.

9. Mattson, S.N. and E.P. Riley, A review of the neurobehavioral deficits in children with fetal alcohol syndrome or prenatal exposure to alcohol. Alcohol Clin Exp Res, 1998; 22(2): 279-94.

10. Mattson, S.N., et al., Neuropsychological comparison of alcohol-exposed children with or without physical features of fetal alcohol syndrome. Neuropsychology, 1998; 12(1): 146-53.

11. Hoyme, H.E., et al., A practical clinical approach to diagnosis of fetal alcohol spectrum disorders: clarification of the 1996 institute of medicine criteria. Pediatrics, 2005; 115(1): 39-47.

12. Roebuck, T.M., S.N. Mattson, and E.P. Riley, A review of the neuroanatomical findings in children with fetal alcohol syndrome or prenatal exposure to alcohol. Alcohol Clin Exp Res, 1998; 22(2):33944. 